\title{
Evaluation of Outcome of Management of Distal Tibial Fractures using Distal Tibial Locking Plate
}

\author{
${ }^{1}$ Mannan Ahmed, ${ }^{2}$ Sanjeev Jindal, ${ }^{3}$ Vivek Bansal, ${ }^{4}$ Rajesh Kapila, ${ }^{5}$ Radhe S Garg
}

\begin{abstract}
Aim: The modern trend in the management of fractures is fast changing in favor of rigid fixation and early mobilization with minimal period of plaster immobilization. We present a prospective cohort study of fixation of distal tibial fractures with distal tibial locking plate and its evaluation in terms of maintenance of accurate anatomical reduction, stable fixation, with early restoration of functions.
\end{abstract}

Materials and methods: A total of 25 patients with median age 38.04 years, age range 19 to 70 , with distal tibial fracture were treated by using distal tibial locking plate. The outcome was evaluated using American Orthopedic Foot and Ankle Society (AOFAS) ankle-hindfoot score.

Results: The majority of fractures in this study were extraarticular (64\%), while $20 \%$ were partial articular fractures and $16 \%$ were complete articular fractures. Out of 25 patients, 6 patients had superficial infection and 1 patient had deep infection. Out of 25 cases, 5 cases had union by 16 weeks, 11 cases by 20 weeks, 7 cases had union by 30 weeks, and 2 cases had nonunion. According to AOFAS score at 6 months, 4 cases had score of 31 to 70 and 21 cases had score of 71 to 100 .

Conclusion: The results of our study corroborate with the contemporary literature relevant to distal tibial fracture fixation performed with various locking plates. Therefore, locking compression plate is a good device to stabilize the fracture of the distal tibial.

Clinical significance: Locking plates are a good device to stabilize the fractures of the distal tibial, especially when used in conjunction with meticulous intraoperative handling of soft tissue and active participation of patients in rehabilitation program. Studying this alternative method expands the present knowledge for the management of distal tibial fractures.

Keywords: American Orthopedic Foot and Ankle Society anklehindfoot score, Arbeitsgemeinschaft Fur Osteosynthesefragen/ Orthopaedic Trauma Association classification, Cohort study, Distal tibial fracture, Distal tibial locking plate.

\footnotetext{
${ }^{1-3}$ Senior Resident, ${ }^{4,5}$ Professor

1,2Department of Orthopaedics, ESI, PGIMSR, Basaidarapur New Delhi, India

${ }^{3}$ Department of Orthopaedics, Maulana Azad Medical College New Delhi, India

${ }^{4,5}$ Department of Orthopaedics, Government Medical College and Hospital, Amritsar, Punjab, India
}

Corresponding Author: Sanjeev Jindal, Senior Resident Department of Orthopaedics, ESI, PGIMSR, Basaidarapur New Delhi, India, Phone: +918059828485, e-mail: jindal_dr@ yahoo.co.in
How to cite this article: Ahmed M, Jindal S, Bansal V, Kapila R, Garg RS. Evaluation of Outcome of Management of Distal Tibial Fractures using Distal Tibial Locking Plate. J Foot Ankle Surg (Asia-Pacific) 2017;4(1):5-9.

Source of support: Nil

Conflict of interest: None

\section{INTRODUCTION}

The evolution of civilization is essentially a record of man's struggle against the adverse environmental changes (Milieu interior and exterior). Although the modern era has seen great many advancements in mechanization and increase in vehicular traffic accidents, road side accidents are becoming epidemiologically one of the largest killers and the most dreadful enemy of human beings all over the world. Today's trauma surgeon is witnessing a sea change in the pattern of injuries in terms of severity, soft-tissue damage, and the accompanying complications.

Fracture of distal tibial is one of the common fractures seen in orthopedic practice following accidents. Because of long period of recumbency and associated socioeconomic burden imposed by these fractures, there have been constant efforts to develop methods of treatment which reduce period of hospitalization and recumbency.

Depending on the fracture pattern, level and soft-tissue damage of the tibia, various methods of management have been described. The indications for operative and nonoperative treatment of tibial fractures continue to be refined. Although commonly advocated in the past, nonoperative treatment now is generally reserved for closed, stable, minimally displaced fractures caused by low-energy trauma. Operative treatment allows early motion, provides soft-tissue access, and avoids complications associated with immobilization. The goals of treatment are to obtain a healed, well-aligned fracture; pain-free weight bearing; and functional range of motion of the knee and ankle joints.

In this study, we present the management of distal tibial fractures using distal tibial locking plate and evaluation of its functional outcome.

\section{MATERIALS AND METHODS}

This prospective study consisted of 25 cases of fractures of distal tibial admitted in the Department of Orthopaedics, Government Medical College and Hospital, Amritsar, Punjab, India. 
Inclusion criteria were Skeletal maturity, fracture of the distal tibial with or without fibular fracture. Exclusion criteria were previous or existing infection in the involved leg and compound grade III fractures where soft-tissue cover to the implant is not possible.

The patients were resuscitated in the emergency room and a complete examination of the patient, for other associated injuries as well, was done. Neurological and vascular assessment of the involved limbs was done. Wound lavage, dressing, and splintage were done as per the initial assessment and injury to the patient. Analgesics, antibiotics, and intravenous fluids were administered as per protocol, and tetanus prophylaxis as per requirement was administered. Basic blood parameters were evaluated. Roentgenograms were assessed to determine the fracture configuration and to plan the operative procedure. Fractures were classified according to Rüedi and Allgöwer system. ${ }^{1}$ The patient was taken for surgery after initial resuscitation, stabilization of vital parameters, and after getting preanesthetic checkup and clearance.

All the patients were operated in supine position on a radiolucent table. Pneumatic tourniquet was applied. The patient was prepared and draped, leaving the leg exposed as required for surgical incision and intraoperative evaluation of fracture alignment.

Once the patient was prepared and draped, immediate preoperative antibiotics were given before the inflation of tourniquet. By traction and manipulation, close reduction was attempted. The provisional reduction was confirmed by image guidance. After adequate reduction and alignment was achieved, plate size was selected under image guidance so as to provide adequate fixation and stabilization of fracture.

If adequate reduction and alignment was achieved, minimally invasive percutaneous plate osteosynthesis (MIPPO) technique was used. If adequate reduction is not achieved, fracture site was exposed by standard medial approach and open reduction and internal fixation was done following standard principles. In case of intraarticular fractures, accurate reduction was confirmed and provisional fixation was done with $\mathrm{K}$ wires or screws before proceeding with plate fixation (Figs 1 to 4 ).

In MIPPO technique, incision was made obliquely at the tip of medial malleolus and extended proximally to create easy passage. The medial malleolus was exposed, with care taken to protect the great saphenous vein. Percutaneous elevators were inserted to create extraperiosteal tunnel for the plate. The passage of the plate till fracture site was confirmed with C-arm imaging. An incision was made proximally at the estimated proximal edge of the plate. The anterior and posterior borders of the medial tibial were palpated and incision was extended longitudinally exposing the periosteum. Submuscular plane was developed in proximal incision and tunnel developed till fracture site and the plate was pushed by the surgeon's opposite hand. The plate was palpated in the proximal incision and confirmed to be well seated. The plate was fixed on the tibial surface with a Kirschner wire inserted through a fixation bolt. Adequate positioning was confirmed with anteroposterior and lateral imaging.

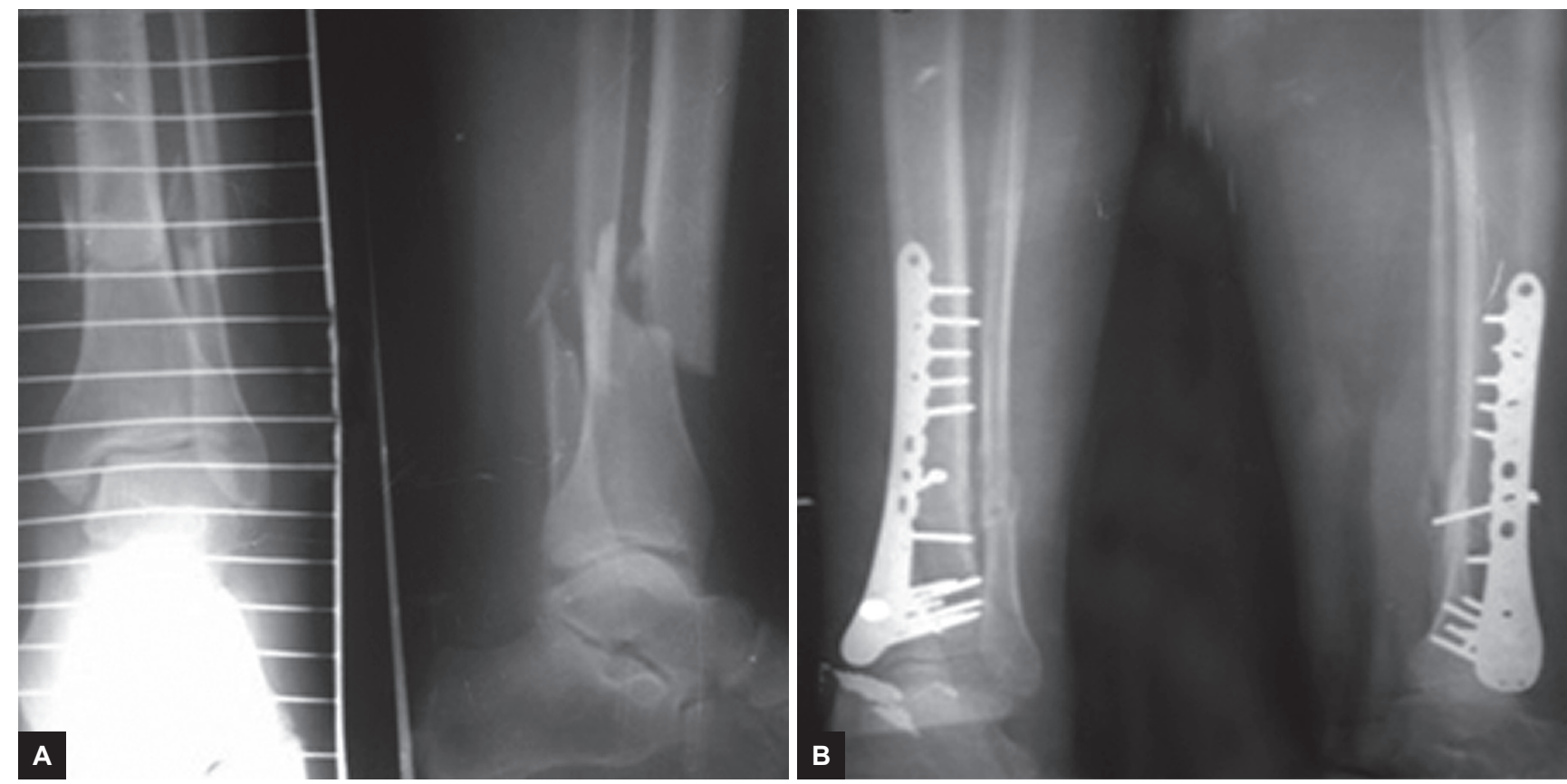

Figs $1 \mathrm{~A}$ and B: (A) Preoperative radiograph of a 40-year-old male patient showing fracture of both leg bones, distal one-third; and $(B)$ postoperative radiograph of the same patient with fracture fixed with distal tibial locking plate 


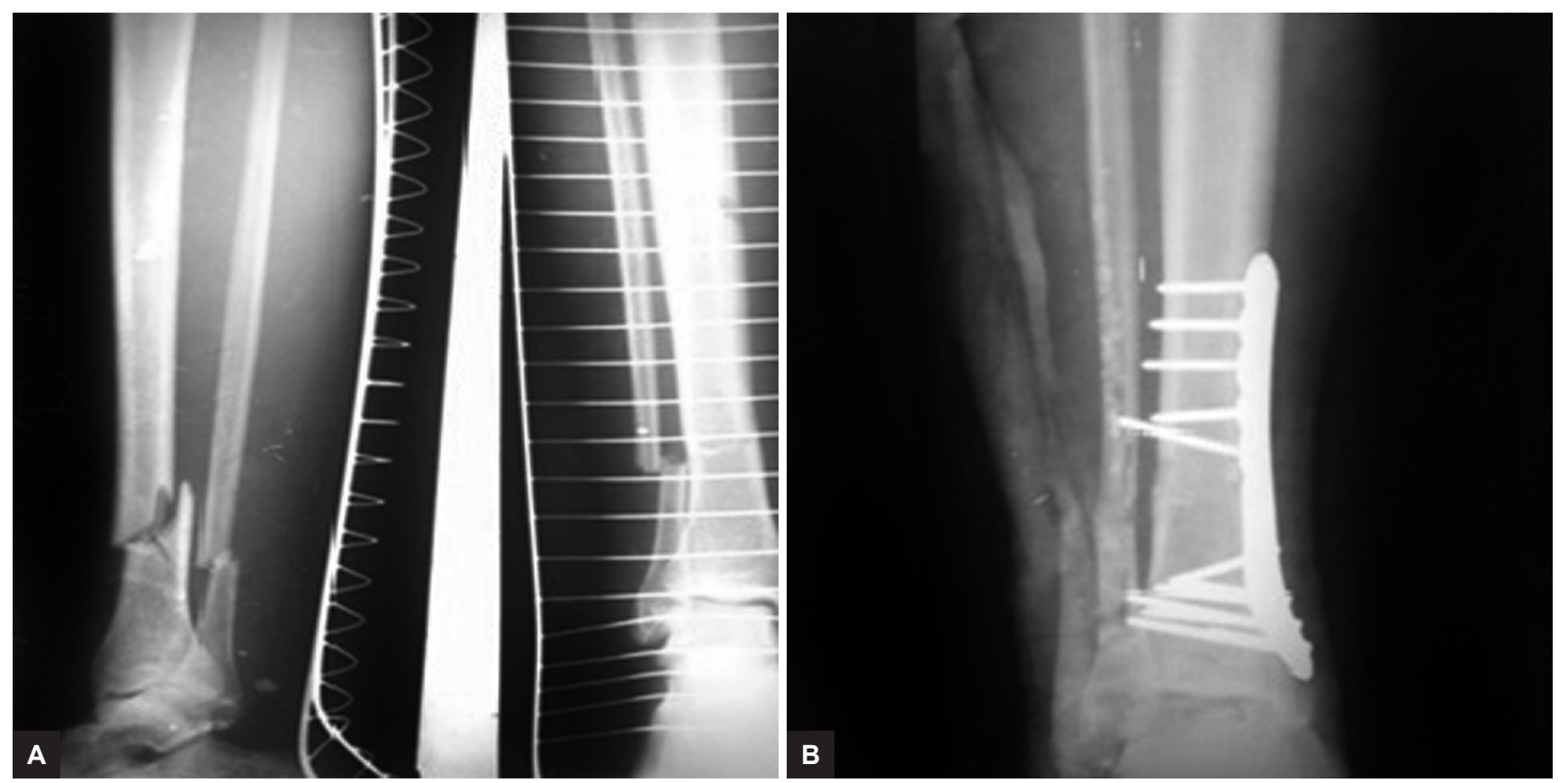

Figs 2A and B: (A) Preoperative radiograph of a 44-year-old male patient showing fracture of both leg bones, distal one-third; and $(B)$ postoperative radiograph of same patient with fracture fixed with distal tibial locking plate
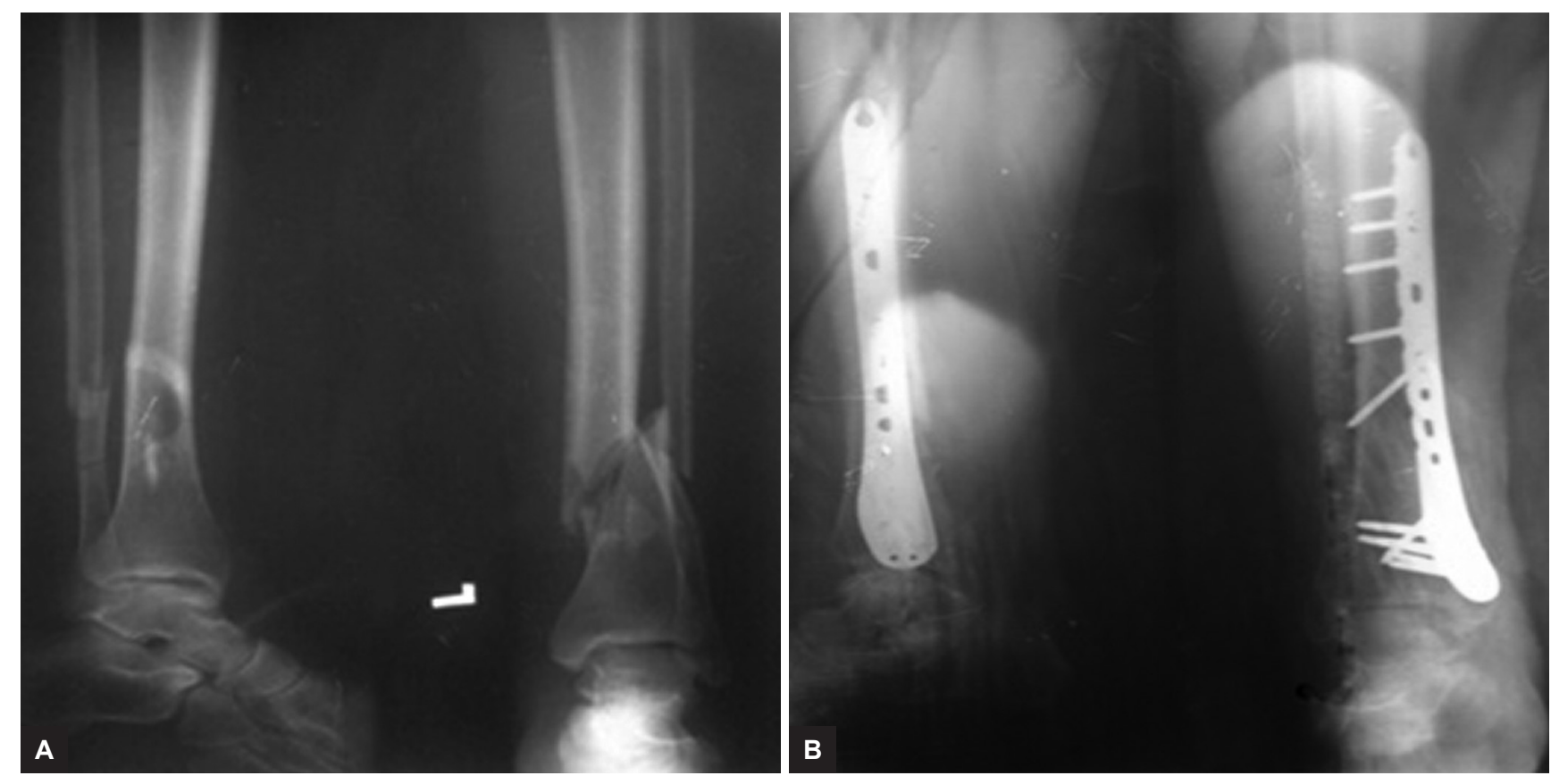

Figs 3A and B: (A) Preoperative radiograph of a 38-year-old male patient showing fracture of both leg bones, distal one-third; and $(B)$ postoperative radiograph of the same patient showing fracture fixed with distal tibial locking plate

This was followed by insertion of fixation screws, following the standard procedure for nonlocking cortical screws and locking screws. All the nonlocking screws were inserted first as decided preoperatively, and after attaining adequate reduction, locking screws were inserted. A minimum of four screws were used in each main fracture fragment. The principles of fixation using locking compression plates (LCP) were adhered to at every stage of fixation. This was followed by irrigation of the incisions with normal saline and wound closure in layers. The technical problems/complications during the procedure (if any) were recorded.

Postoperative X-ray was done to document proper reduction and fixation of fracture fragments. Ankle mobilization was started as early as possible depending upon condition of patient and level of fixation. Antibiotics (intravenous/oral) were continued till the wound condition necessitates.

Regular follow-up of the patient in outpatient department with X-rays and functional outcome evaluated as per 


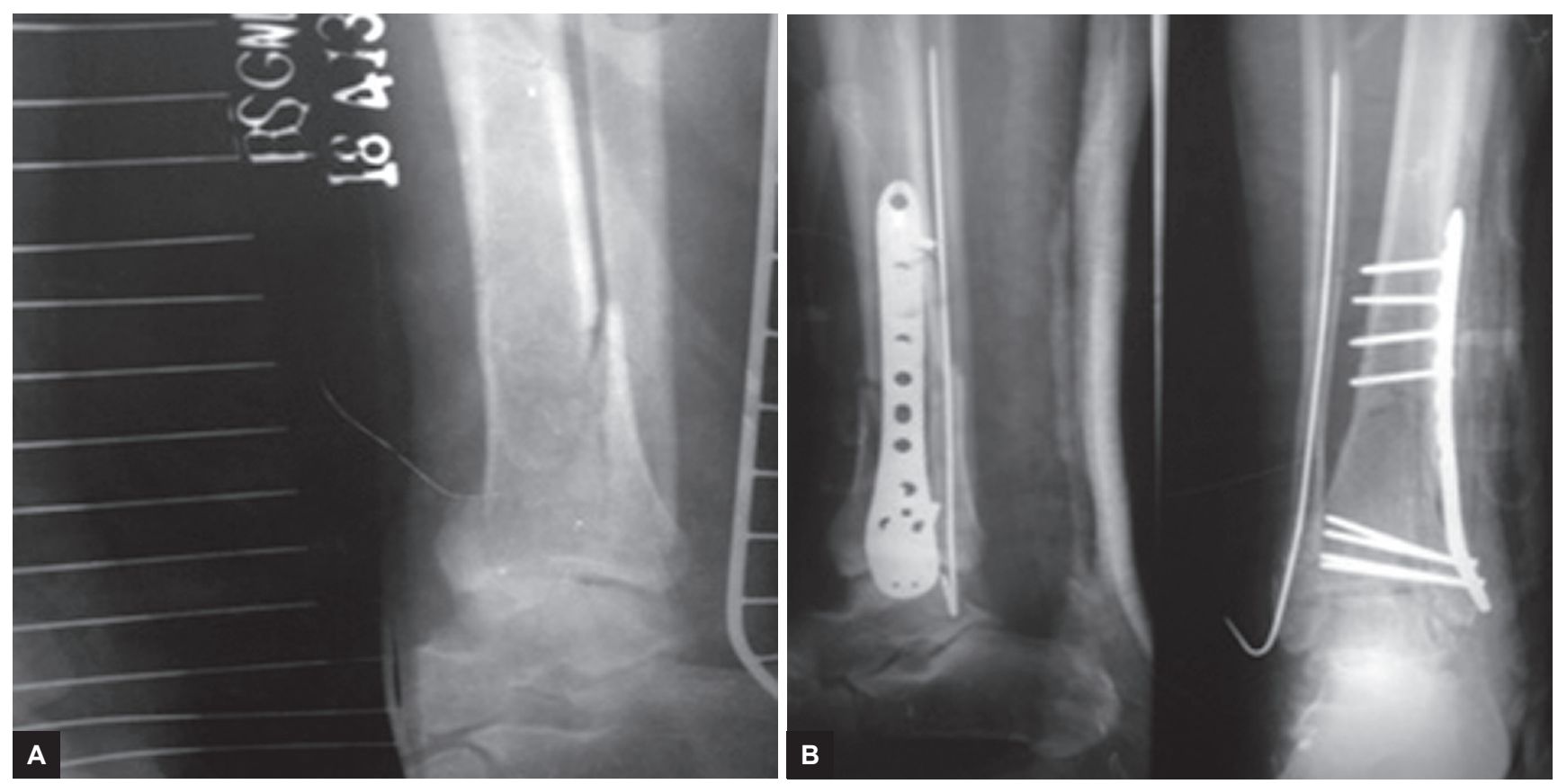

Figs 4A and B: (A) Preoperative radiograph of a 30-year-old male patient showing fracture of both leg bones, distal one-third; and $(B)$ postoperative radiograph of the same patient with fracture fixed with distal tibial locking plate

ankle-hindfoot scoring system [American Orthopedic Foot and Ankle Society (AOFAS) score] was done. ${ }^{2}$ All longterm complications like nonunion, malunion, angular deformity, implant breakage, shortening, or infection were recorded. The patient was under follow-up till the bony union of the fracture/up to 6 months, whichever is earlier. The results were compiled and compared with the literature. If any complications occur during operation or postoperatively, they were managed accordingly. The outcome of distal tibial fracture management with locking plates was assessed in terms of time to bony union, ankle range of movement, infection, secondary procedures performed, and implant failure. The data so generated were evaluated and expressed as descriptive statistics.

\section{ETHICAL AND HUMANE CONSIDERATIONS}

All patients gave written informed consent to be included in this study, and the study was authorized by the local ethical committee carried out in accordance with the World Medical Association Declaration of Helsinki (Journal of Bone and Joint Surgery, 1997 Jul;79-A:1089-1098).

\section{OBSERVATION AND RESULTS}

The present study enrolled 25 patients with fracture of distal end tibial and average age of 38.04 (19-70) years. It was observed that comminuted fractures of the distal end tibial were more common in young and middle-age groups, with higher incidence in 3rd and 4th decade of life ( $52 \%$ patients between 31350 years of age). In our series, 25 patients were analyzed, of whom 19 were males and
6 were females (male:female ratio 3.16:1), so there was higher incidence of these injuries in males. Table 1 shows distribution of patients according to age.

Road traffic accident was the most common cause of injury in this study. Sixteen patients suffered road traffic accident, 7 sustained it after a domestic fall, and 2 due to assault. Out of seven patients who sustained injury after fall, four were females and three were males. Among the 25 patients analyzed in this study, 9 patients had associated injuries, 3 cases with head injury, 1 case with chest injury, 1 with fracture patella and medial malleolus, 1 patient had nasal bone fracture, 1 patient with fracture patella, 1 case with avulsion over opposite leg, and 1 case with fracture of the ulna.

The 25 distal tibial fractures analyzed in this study were graded in accordance to the Arbeitsgemeinschaft Fur Osteosynthesefragen/Orthopaedic Trauma Association classification. ${ }^{3}$ Majority of fractures in this study were extraarticular (64\%), while $20 \%$ were partial articular fractures, and $16 \%$ were complete articular fractures. Table 2 shows distribution according to the type of fracture. Table 3 shows distribution according to type

Table 1: Distribution of subjects according to age

\begin{tabular}{lll}
\hline Age (years) & No. of patients & $\begin{array}{l}\text { Percentage } \\
\text { of patients }\end{array}$ \\
\hline $19-30$ & 7 & 28 \\
$31-40$ & 8 & 32 \\
$41-50$ & 5 & 20 \\
$51-60$ & 4 & 16 \\
$61-70$ & 1 & 4 \\
\hline
\end{tabular}


Evaluation of Outcome of Management of Distal Tibial Fractures using Distal Tibial Locking Plate

Table 2: Distribution of subjects according to type of fracture

\begin{tabular}{lll}
\hline Type of fracture & No. of patients & $\begin{array}{l}\text { Percentage } \\
\text { of patients }\end{array}$ \\
\hline A & 16 & 64 \\
B & 5 & 20 \\
C & 4 & 16 \\
\hline
\end{tabular}

Table 4: Distribution of subjects according to time to bony union

\begin{tabular}{lll}
\hline Time & No. of patients & $\begin{array}{l}\text { Percentage } \\
\text { of patients }\end{array}$ \\
\hline By 16 weeks & 5 & 20 \\
By 20 weeks & 11 & 44 \\
By 30 weeks & 7 & 28 \\
Nonunion & 2 & 8 \\
\hline
\end{tabular}

of infection. Out of 25 cases, 2 cases required debridement and 1 case required debridement and implant removal.

Out of 25 cases, 5 cases had union by 16 weeks, 11 cases by 20 weeks, 7 cases had union by 30 weeks, and 2 cases had nonunion. Two cases had malunion of fracture. According to AOFAS score at 6 months, 4 cases had score of 31 to 70 and 21 cases had score of 71 to 100 . Tables 4 and 5 show distribution according to time to union and total AOFAS score respectively.

\section{DISCUSSION}

Distal tibial fractures have always posed a challenge to treatment, especially when associated with soft-tissue injury and comminution. In the present study, AOFAS ankle and hindfoot score was applied to analyze the functional outcome among the cases. After evaluation, it was observed that the average AOFAS score at 6 months was 85.3.

In a prospective study of 42 patients, reviewed at a mean of 19.6 months after treatment of distal tibial and pilon fractures using the AO distal tibial locking plate, mean AOFAS score was 90 at a mean of 19 months follow-up. ${ }^{4}$

In another prospective study performed on 13 patients with tibial fractures treated with indirect reduction and minimally invasive percutaneous LCP internal fixation, according to the AOFAS score, the function of the ankle joint was graded 80 to 95 (92.4 on average). ${ }^{5}$

A prospective study evaluated clinical results and outcomes of low metaphyseal distal tibial fractures. These were treated using the minimally invasive plate osteosynthesis concept with a $3.5 \mathrm{~mm}$ locked medial tibial plafond plate and hybrid (locking and nonlocking) screw construct. Thirty-eight patients were followed on average for 32 months (12-48 months). In 30 patients at 2 years, the AOFAS and the Olerud and Molander ${ }^{6}$ ankle scores averaged good to excellent. ${ }^{7}$

Hence, the result of our study corroborates with the contemporary literature relevant to distal tibial fracture
Table 3: Distribution of subjects according to type of infection

\begin{tabular}{lll}
\hline Infection & No. of patients & $\begin{array}{l}\text { Percentage } \\
\text { of patients }\end{array}$ \\
\hline Superficial & 6 & 24 \\
Deep & 1 & 4 \\
Absent & 18 & 72 \\
\hline
\end{tabular}

Table 5: Distribution of subjects according to total score (AOFAS score)

\begin{tabular}{lllll}
\hline Score & 1 month & 2 months & 3 months & 6 months \\
\hline $0-30$ & 11 & 4 & 2 & 0 \\
$31-70$ & 14 & 19 & 18 & 4 \\
$71-100$ & 0 & 2 & 5 & 21 \\
\hline
\end{tabular}

fixation performed with various locking plates. This can be attributed to preoperative antibiotic coverage, early surgical intervention, accurate reduction, meticulous aseptic technique, and early postoperative active exercises.

\section{CONCLUSION}

Locking plates are a good device to stabilize the fractures of the distal tibia, especially when used in conjunction with meticulous intraoperative handling of soft tissue and active participation of patients in rehabilitation program. But at the same time, it is highly unforgiving device if the principles of LCP and technicalities of procedure are not strictly followed. We shall add a word of caution; LCPs are specialized implants, to be used with careful preoperative planning and meticulous surgical procedure.

\section{REFERENCES}

1. Rüedi TP, Allgöwer M, Fractures of the lower end of the tibia into the ankle joint. Injury 1969 Oct;1(2):92-99.

2. Kitaoka HB, Alexander IJ, Adelaar RS, Nunley JA, Myerson MS, Sanders M. Clinical rating systems for the ankle-hindfoot, midfoot, hallux, and lesser toes. Foot Ankle Int 1994 Jul;15(7):349-353.

3. Orthopaedic Trauma Association Committee for Coding and Classification. Fracture and dislocation compendium. J Orthop Trauma 1996;10(suppl 1):1-154.

4. Bahari S, Lenehan B, Khan H, McElwain JP. Minimally invasive percutaneous plate fixation of distal tibia fractures. Acta Orthop Belg 2007 Oct;73(5):635-640.

5. Zha G, Chen Z, Qi X. Minimally invasive percutaneous locking compression plate internal fixation in the treatment of tibial fractures. Zhongguo Xiu Chong Jian Wai Ke Za Zhi 2008 Dec;22(12):1448-1450.

6. Olerud C, Molander H. A scoring scale for symptom evaluation after ankle fracture. Arch Orthop Trauma Surg 1984;103(3):190-194.

7. Collinge C, Kuper M, Larson K, Protzman R. Minimally invasive plating of high-energy metaphyseal distal tibia fractures. J Orthop Trauma 2007 Jul;21(6):355-361. 